\title{
$\underline{\mathbf{P}-121}$
}

\section{The Use of Orthosiphon Stamineus Anticancer Action Against Human Oral Squamous Cell Carcinoma}

\author{
Luay Thanoon Younis*, Mohamed Ibrshim Abu Hassan, Fara Fariza Zahar and Mohd Faizul Mohd \\ Husin \\ Centre of Studies for PreClinical Sciences, Faculty of Dentistry, Universiti Teknologi MARA, 40450 Shah Alam, \\ Selangor Darul Ehsan; E-mail: drluay@salam.uitm.uitm.edu.my
}

Background: Oral squamous cell carcinoma (OSCC) accounts about $90 \%$ of head and neck cancer. Orthosiphon stamineus is a medicinal herb grown in Southeast Asia and exhibit many pharmacological properties such as, anti-inflammatory, antioxidant, anti-bacterial, antiangiogenetic effects.

Objective: To investigate the anticancer effect of Orthosiphon stamineus against human OSCC in vitro.

Methods: The proliferative activity of the OSCC was investigated by the doubling time assay. Proliferation essay was done to assess the anti-proliferative effect of Orthosiphon stamineus aqueous extract by treating the human OSCC with $0-300 \mu \mathrm{g} / \mathrm{ml}$ of the aqueous extract. The efficacy of Orthosiphon stamineus to inhibit the recovery of the OSCC colonies was measured by clonogenic assay. Caspase-3 activity was investigated using colorimetric assay.

Results: The results obtained from the human tumor colony-forming assay showed that Orthosiphon stamineus has suppressed the recovery of OSCC colonies in dose-dependant manner. Orthosiphon stamineus inhibits the clonogenic potential of OSCC with minimal cell surviving fraction of 0.0015 . The surviving percentage of the proliferating OSCC decreases by increasing the concentration of the extract. Caspase activity was also enhanced by Orthosiphon stamineus.

Conclusion: The use of Orthosiphon stamineus may be beneficial for the suppression of the human OSCC and it may serve as a chemopreventive or therapeutic agent for the oral cancer.

Keywords: Orthosiphon stamineus, OSCC, Anti-cancer. 